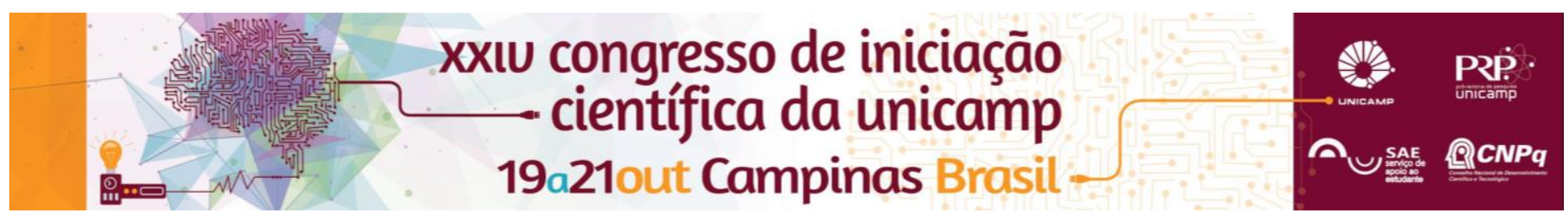

\title{
Development of a computational tool to investigate supersonic flows in rocket nozzles.
}

\author{
Frederico R. Abreu*, William R. Wolf
}

\begin{abstract}
This work is based on the analysis of the physical phenomena that occurs in the supersonic flow on a rocket nozzle. We develop a computational tool which solves the 2D Euler equations in integral form. A finite volume method is employed in the numerical simulations where the Steger and Warming upwind scheme is implemented for the spatial discretization and a first-order Runge Kutta scheme is applied for the time integration of the non-linear set of coupled equations. Boundary conditions are implemented representing a supersonic inlet and outlet of the nozzle and slip conditions are used along the nozzle walls. The computational tool is able to capture the shockwaves and expansion waves formed inside the nozzle including their interactions. The computational tool was previously validated for the supersonic flow past a wedge configuration. The numerical tool will serve as the foundation to future work which will include the optimization of the nozzle geometry for reduced wave drag and for the design of efficient supersonic vehicles.
\end{abstract}

\section{Key words:}

CFD, supersonic flow, upwind scheme.

\section{Introduction}

With the fast development of technology, new and faster ways to travel have been studied. Recently, a new generation of supersonic engines have been studied as an alternative: the scram jets. These supersonic engines can reach hypersonic speeds (beyond Mach 5) ${ }^{1}$. The present research has the objective to study the flow physics that occurs in supersonic flows including shock waves, expansion waves and their interactions. A rocket nozzle configuration is analyzed using a computational tool which solves the 2D Euler equations in integral form using a finite volume method. The numerical formulation employs the Steger and Warming upwind scheme ${ }^{2}$ for the spatial discretization. This scheme solves the Euler equations through an adequate splitting of the flux vectors. Here, the fluxes are written in terms of the Jacobian matrices and the conserved flow variables. The Jacobian matrices are diagonalized and the eigenvalues are selected based on the direction of the propagation of acoustic and entropy waves, following the Riemman invariants.

\section{Results and Discussion}

The present work solves the 2D Euler's equations as the model for the supersonic flow in the nozzle. Therefore, viscosity and heat transfer are neglected in the solution. In order to reach a steady state solution of the Euler equations, the finite volume approach is integrated in time using a Runge-Kutta scheme. The formation and reflection of shockwaves inside the nozzle are well captured as it can be seen by the discontinuities along the convergent stage shown on Images 1 and 2 , as well as the effect of the interaction among them, which caused another abrupt change on the flow properties. The expansion waves are also captured, as shown on Images 1 and 2 by the smooth changes in Mach number and density along the expansion stage of the nozzle.
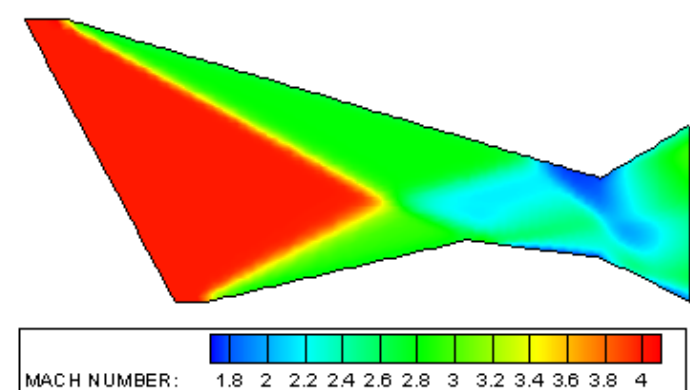

Image 1: Mach number contours along the nozzle.

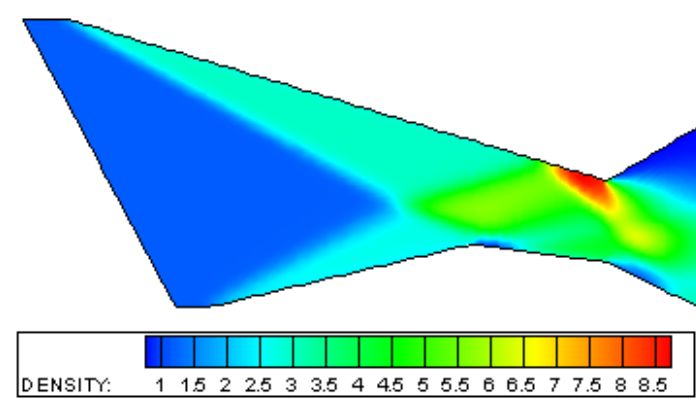

Image 2: Density contours along the nozzle.

\section{Conclusions and Future Work}

A numerical tool was developed to solve supersonic flows inside rocket nozzles. The numerical methodology was validated for simpler flow configurations. A first order upwind method was employed and showed excellent results, capturing shock and expansion waves, and their interactions. The numerical tool will be used for optimization studies of hypersonic vehicles solving the adjoint approach.

\footnotetext{
1 NASA Armstrong Flight Research Center webpage, http://www.nasa.gov/centers/armstrong/news/FactSheets/FS-040-DFRC.html (accessed Jun, 13h, 2015)

2 Anderson, J. D., Computational Fluid Dynamics: the basics with applications, McGraw-Hill, USA, 1995, 563p.
} 